\title{
Ocular hypertension after intravitreal triamcinolone with vitrectomy and phacoemulsification
}

This article was published in the following Dove Press journal:

Clinical Ophthalmology

16 June 2012

Number of times this article has been viewed

\author{
D Wilkin Parke III \\ Robert A Sisk \\ Samuel K Houston \\ Timothy G Murray \\ Department of Ophthalmology, \\ Bascom Palmer Eye Institute, \\ University of Miami, Miller School \\ of Medicine, Miami, FL, USA
}

Correspondence: Timothy G Murray Bascom Palmer Eye Institute, 900 NW

17th St, Miami, FL 33136, USA

$\mathrm{Tel}+\mathrm{I} 3053266000$

$\mathrm{Fax}+\mathrm{I} 3053266417$

Email tmurray@med.miami.edu
Objective: To evaluate the effect of adjunctive intravitreal triamcinolone (IVTA) on intraocular pressure (IOP) in the setting of combined vitrectomy with phacoemulsification.

Design: Retrospective case series.

Participants: One hundred thirty-one consecutive eyes undergoing nonemergent vitrectomy with phacoemulsification and IVTA were reviewed and included in the analysis. All 131 eyes received $4 \mathrm{mg}$ IVTA at the end of surgery.

Methods: Pre- and postoperative IOP, use of pressure-lowering medications, and rate of glaucoma surgery were analyzed. Pre-operative risk factors were analyzed.

Main outcome measures: IOP, glaucoma medications, or glaucoma surgery.

Results: Secondary ocular hypertension (defined as IOP $\geq 25 \mathrm{mmHg}$ ) was found in 28 eyes (21\%), the majority during postoperative day 1 only. Twelve eyes (9\%) had an elevated IOP measurement noted at a visit after the first postoperative day. Five (4\%) had an IOP rise of $\geq 10 \mathrm{mmHg}$ over baseline at any time after postoperative day 1 . Six (5\%) required glaucoma medications. One eye required a glaucoma drainage implant for diabetic neovascular angle closure glaucoma, and one eye required enucleation for intractable neovascular glaucoma due to radiation retinopathy. Elevated postoperative IOP was statistically associated with higher baseline IOP and presence of preoperative glaucoma.

Conclusions: Therapeutic intravitreal triamcinolone with combined vitrectomy and phacoemulsification causes infrequent and usually mild secondary ocular hypertension. Secondary ocular hypertension is associated with preoperative glaucoma and high IOP.

Keywords: glaucoma, cataract, inflammation

\section{Introduction}

The pressure-related effect of intravitreal steroid injection has been well-characterized in the nonvitrectomized eye. ${ }^{1-4}$ Intravitreal triamcinolone may be associated with a more muted effect on intraocular pressure (IOP) in vitrectomized eyes, but the incidence and severity of secondary ocular hypertension in these eyes is still unclear. ${ }^{5,6}$ Even less is known about the pressure effect of triamcinolone when used as therapeutic surgical adjunct at time of vitrectomy. Vitrectomy surgery itself has been implicated recently as a precipitant of secondary open angle glaucoma, particularly in nonphakic eyes. ${ }^{7,8}$

Intravitreal triamcinolone (IVTA) has the potential to limit postoperative inflammation and cystoid macular edema in patients undergoing vitrectomy. A combination of vitrectomy and phacoemulsification is becoming an increasingly 
common means of addressing vitreoretinal pathology and cataract while limiting operative exposure. Cataract extraction at time of vitrectomy can improve the intra- and postoperative view to the posterior pole, accelerate visual rehabilitation, and eliminate the risks and costs inherent with a subsequent phacoemulsification procedure in a vitrectomized eye. Several series have shown comparative improvement in postoperative visual acuity when vitrectomy was combined with phacoemulsification, with no associated rise in complication frequency. ${ }^{9-12}$ The inflammatory response can be greater with combined surgery, however, and intraoperative agents such as triamcinolone have garnered recent interest for the prevention of postoperative inflammation.

This retrospective, consecutive case series presents intraocular pressure analysis in eyes that underwent combined phacoemulsification and vitrectomy with intravitreal triamcinolone acetonide. The primary objective was to analyze the incidence of glaucoma after surgery.

\section{Patients and methods}

The study was performed with the approval of the University of Miami Institutional Review Board and in accordance with the US Health Insurance Portability and Accountability Act and the Declaration of Helsinki guidelines. A retrospective review was conducted of all patients who underwent combined, sutureless, 23-gauge pars plana vitrectomy with phacoemulsification cataract extraction and IVTA injection between January 1, 2006, and May 30, 2009, at the Bascom Palmer Eye Institute, by a single vitreoretinal and ocular oncology surgeon (TGM) and his surgical fellows.

All the patients had more than 6 months of follow-up. Four eyes were excluded due to postoperative retinal detachments that could affect IOP, and one was excluded because of persistent postoperative hypotony. A total of 131 eyes met inclusionary criteria and were included in the analysis. Medical records were reviewed and data collected regarding demographics, preoperative ocular and systemic disease, prior intraocular injections or laser treatment, prior ocular surgery, indications for surgery, type of surgery, postoperative course, and any secondary surgeries. All patients had a clinically significant cataract at the time of surgery.

Surgery was performed with the Accurus 2500 surgical system (Alcon Laboratories, Fort Worth, TX). Preoperative intraocular lens biometry was performed with the IOL Master (Carl Zeiss Meditech, Dublin, CA). Postoperative clinic evaluations were performed at 1 day, 1 week, and 1, 3, 6, and 12 months. Patients received topical anti-inflammatory, antibiotic, and cycloplegic drops postoperatively when indicated.

Patients received a single injection of $4 \mathrm{mg}$ in $0.1 \mathrm{~mL}$ of triamcinolone acetonide (Triesence; Alcon Laboratories, Fort Worth, TX) at the time of surgery. The injection was performed through the pars plana into the vitreous cavity with a 27-gauge needle after the vitrectomy and removal of instrumentation and cannulas. Gentamicin $(20 \mathrm{mg})$ and dexamethasone $(4 \mathrm{mg})$ were injected subconjunctivally at the end of the procedure.

Intraocular pressure was measured by Goldmann applanation preoperatively and at day 1 , week 1 , and months 1, 3, 6, and 12 after surgery. Glaucoma medication and surgery usage was documented.

\section{Statistical analysis}

Analysis was performed using the paired and unpaired Student's $t$-tests for continuous variables and the Fisher's exact test for $2 \times 2$ tables of categorical data, as appropriate. Excel 2007 software (Microsoft, Redmond, WA) was used for statistical analysis. A $P$-value of less than 0.05 was considered statistically significant.

\section{Results}

Table 1 lists the surgical indications for the cohort. During the study period, 131 eyes from 131 patients underwent combined 23-gauge sutureless vitrectomy with phacoemulsification and IVTA for various indications and were included in the analysis.

Mean age at surgery was 59.5 years, and mean follow-up was 337 days (range, 174-645 days). All eyes had recorded IOP preoperatively and at day 1 and months 1,3 , and 6 postoperatively. One hundred five eyes (80\%) had a postoperative visit and IOP measurement at month 12. All eyes were phakic preoperatively. Three eyes were in diabetic patients, and two eyes had previously undergone vitrectomy. Primary vitreoretinal indications for the surgery were diverse and reflected an oncologic service at a busy academic center. Of note, twelve eyes had noted optic nerve compromise from prior radiation optic neuropathy. Surgery variably included membrane peeling, endolaser, fluid-air exchange, gas tamponade, and silicone oil removal. Eighty-one eyes $(62 \%)$ went on to receive subsequent intravitreal injections within the study follow-up period; 75 received bevacizumab or ranibizumab, and six received IVTA. None of the six patients who received additional doses of IVTA developed high IOP, and none of the additional IVTA injections were before the postoperative month 3 visit (mean, 5 months after surgery). 
Table I Characteristics of patients with and without elevated IOP ( $\geq 25 \mathrm{mmHg}$ ) after IVTA with vitrectomy and phacoemulsification

\begin{tabular}{|c|c|c|c|c|}
\hline & All & Elevated postoperative IOP* & No elevated postoperative IOP & $P$ value \\
\hline Number & $13 \mid$ & 12 & 119 & \\
\hline Mean age (years) $\pm S D$ & $61.9 \pm 15.3$ & $63.2 \pm 13.7$ & $61.8 \pm 15.6$ & 0.77 \\
\hline Mean baseline IOP $(\mathrm{mmHg}) \pm$ SD & $15.7 \pm 5.1$ & $21.8 \pm 10.4$ & $15.1 \pm 3.8$ & $<0.000$ I \\
\hline Female (\%) & $81(62)$ & $5(42)$ & $74(62)$ & 0.21 \\
\hline Prior vitrectomy (\%) & $8(6.1)$ & I (8.3) & $7(5.9)$ & 0.55 \\
\hline Preexisting glaucoma (\%) & $19(14.5)$ & $6(50)$ & $13(10.9)$ & 0.0023 \\
\hline Preexisting diabetes (\%) & $3(2.3)$ & I (8.3) & $2(1.7)$ & 0.25 \\
\hline
\end{tabular}

Note: *Excluding IOP value on postoperative day I.

Abbreviations: IVTA, intravitreal triamcinolone; IOP, intraocular pressure; SD, standard deviation.

The mean preoperative IOP was 15.7 (range 9-52, SD 5.11). Fifteen eyes (11\%) had a preoperative IOP $\geq 21 \mathrm{mmHg}$ and five $(4 \%)$ had a preoperative IOP $\geq 25 \mathrm{mmHg}$. Nineteen eyes (15\%) were on a preoperative glaucoma drop (mean 2.4 drops per patient). Of note, one patient had a preoperative IOP of $52 \mathrm{mmHg}$ due to neovascular glaucoma secondary to a central retinal vein occlusion.

Mean IOP was 17.4 on postoperative day 1 (range 4-44), 15.4 at postoperative month 1 (range $8-47$ ), 14.4 at postoperative month 3 (range 6-34), 14.5 at postoperative month 6 (range 9-30), and 15.2 at postoperative month 12 (range 7-32) (Figure 1).

Twenty-eight eyes ( $21 \%$ ) had an IOP $\geq 25 \mathrm{mmHg}$ noted postoperatively; the majority - 16 eyes $(12 \%)$ - were on postoperative day 1 only. IOP normalized after day 1 in this group. Postoperative day 1 IOP was likely affected more by the surgical procedure, expansile gas or fluid usage, residual viscoelastic, and wound management than steroid response; therefore, these eyes were not included in further analysis of those with postoperative hypertension.
Twelve eyes (9\%) developed secondary ocular hypertension, or an IOP $\geq 25 \mathrm{mmHg}$, at some point postoperatively (outside of postoperative day 1). Of those 12 eyes, the IOP elevation was first noted at month 1 in six eyes $(50 \%)$, month 3 in five eyes (42\%), and month 12 in one eye (8\%) (Figure 2). By 3 months postoperatively, eleven eyes had a documented visit with elevated IOP. Secondary ocular hypertension was statistically correlated with higher baseline IOP $(P<0.0001)$ and pre-existing glaucoma $(P=0.0023)$ (Table 2).

Change in IOP from baseline preoperative value was also evaluated. A rise of $\geq 10 \mathrm{mmHg}$ over preoperative IOP was considered significant. Excluding postoperative day 1 values, this rise of $10 \mathrm{mmHg}$ occurred in seven patients (5\%). It was noted at month 1 in two eyes, both of which had neovascular glaucoma (discussed later). It was noted at month 3 in four eyes, and month 12 in one eye. The four eyes that developed a rise of $\geq 10 \mathrm{mmHg}$ at 3 months had a mean IOP of 25 (range 22-27); they were treated with glaucoma drops, and their mean IOP at month 12 was 20.8 (range 16-23).

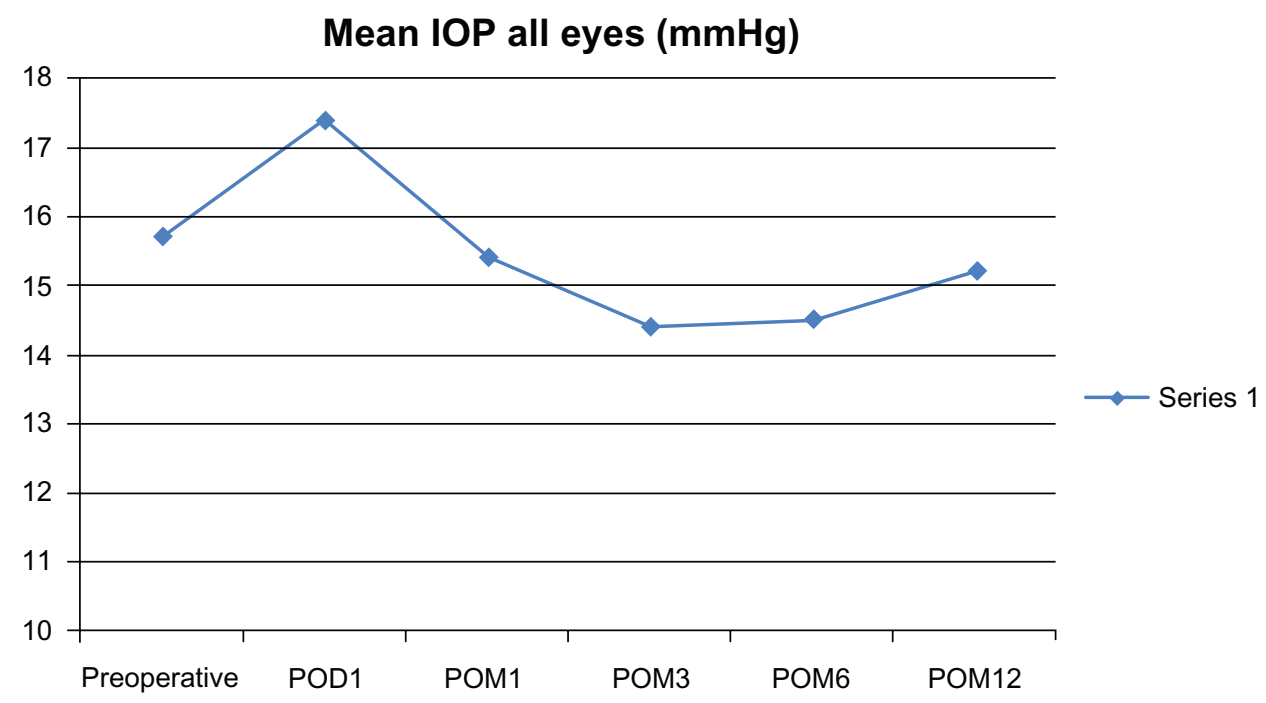

Figure I Mean IOP over time.

Abbreviations: IOP, intraocular pressure; POD, postoperative day; POM, postoperative month. 


\section{Number of eyes with IOP $>25 \mathrm{mmHg}$ (\% of total eyes)}

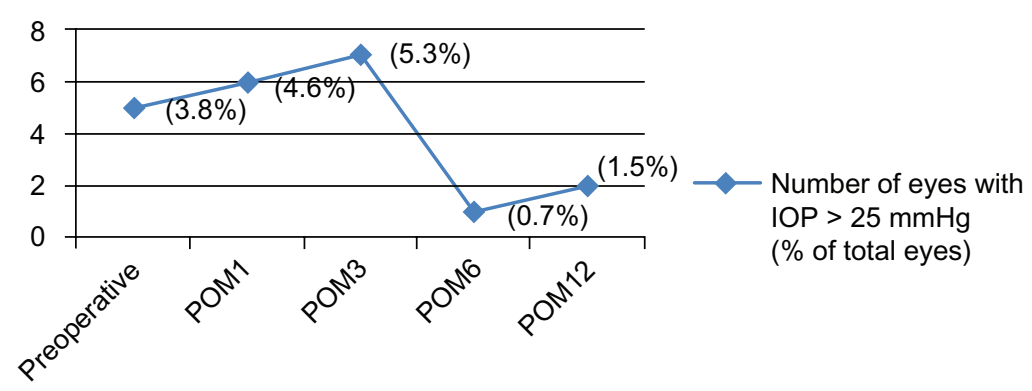

Figure 2 Eyes with IOP over $25 \mathrm{mmHg}$.

Abbreviations: IOP, intraocular pressure; POM, postoperative month.

The one eye with IOP rise $\geq 10 \mathrm{mmHg}$ at month 12 had an IOP of 32 at that visit. Two glaucoma drops were added, and the IOP decreased to 18 at month 18 .

In the eyes with IOP $\geq 25 \mathrm{mmHg}$, the peak pressure ranged from 25 to 47 , with a mean of 31.6. The mean increase of peak IOP over baseline was 9.8 (range 0-32). Among these eyes, the number with increase over baseline of $1-5 \mathrm{mmHg}$, 6-10 $\mathrm{mmHg}, 11-15 \mathrm{mmHg}$, and 16-20 mmHg were two (17\%), four (33\%), two (17\%), and two (7\%), respectively. Six

Table 2 Primary indication for vitrectomy (all patients with clinically significant cataract)

\begin{tabular}{ll}
\hline Indication & Number (\%) \\
\hline Radiation retinopathy with refractory cystoid & 34 \\
macular edema or neovascularization & \\
Primary uveal malignant melanoma & II \\
Epiretinal membrane & 10 \\
Recurrent retinal detachment & 8 \\
Exudative macular degeneration & 8 \\
Intractable subretinal hemorrhage & 5 \\
Solitary choroidal hemangioma & 5 \\
Diffuse choroidal hemangioma & 4 \\
Retinal capillary hemangioma & 4 \\
Biopsy of iris or choroidal mass & 4 \\
Tractional retinal detachment & 3 \\
Uveal effusion & 3 \\
Nonclearing vitreous hemorrhage & 3 \\
Primary intraocular lymphoma & 3 \\
Cataract with unstable zonules & 2 \\
Macular hole & 2 \\
Myopic retinoschisis & 2 \\
Proliferative diabetic retinopathy & 2 \\
Retained silicone oil or perfluorocarbon liquid & 2 \\
Central serous retinopathy & 2 \\
Neovascular glaucoma with vitreous hemorrhage & 2 \\
Dense vitreous opacities & 2 \\
Idiopathic polypoidal choroidal vasculopathy & 2 \\
Coats disease & 2 \\
Treated retinoblastoma & 2 \\
\hline & 2 \\
\hline
\end{tabular}

eyes $(50 \%)$ with postoperative secondary hypertension had pre-existing glaucoma and were already on glaucoma drops; two of these required an additional drop postoperatively. Six eyes $(50 \%)$ were not on pre-existing glaucoma therapy, three of which required initiation of glaucoma drops. The ones who were never started on therapy all had a single IOP measurement in the $25-27 \mathrm{mmHg}$ range that returned to normal at subsequent visits. All eyes had optic nerve examinations at each postoperative visit, and no eyes demonstrated clinical glaucomatous progression of the optic nerve during the follow-up period.

Regarding further interventions, one eye with proliferative diabetic retinopathy had an IOP of $47 \mathrm{mmHg}$ at postoperative month 1 , with chronic synechial angle closure, and required a Baerveldt glaucoma drainage device at week 6 postoperatively. The surgical indication for the vitrectomy had been proliferative retinopathy with refractory diabetic macular edema. One other eye developed intractable neovascular glaucoma secondary to severe radiation retinopathy and ultimately required enucleation. No other eyes went to glaucoma surgery during known follow-up.

\section{Discussion}

The combination of vitrectomy and phacoemulsification is increasingly common and reduces the surgical burden for patients with vitreoretinal disease in conjunction with cataract. It enhances visualization during the vitrectomy portion of the surgery when there is a clinically significant lens opacity, and it can speed visual rehabilitation postoperatively. Both procedures elicit inflammation, however, and the combination of the two may further exacerbate it. ${ }^{9-13}$ Cystoid macular edema is one such inflammatory response that can compromise visual outcome. Utilization of anti-inflammatory agents in the postoperative period is pervasive for both 
vitreoretinal and cataract surgery, usually in topical or subconjunctival form. IVTA injection at the end of surgery offers a more direct and rapid means of delivering antiinflammatory effect to the posterior segment. Triamcinolone has been associated with anti-inflammatory, neuroprotective, and antivascular endothelial growth factor effects in the retina, all of which may contribute to a reduction in cystoid macular edema. ${ }^{14-16}$

Glaucoma and cataracts are the two most common consequences of intra- or periocular steroid usage. Cataract formation secondary to IVTA was not relevant to this study, as all eyes received phacoemulsification at the time of IVTA injection. Steroid-related pressure rise and secondary glaucoma were scrutinized, as glaucoma remains a significant adverse effect and concern among treating physicians. In the safety analysis for the SCORE Study, 35\% of eyes receiving $4 \mathrm{mg}$ of triamcinolone were started on IOP-lowering medications within 12 months of injection, compared to $20 \%$ in the $1 \mathrm{mg}$ triamcinolone group, and $8 \%$ in the control group. ${ }^{17}$ No eyes in that study required glaucoma surgery because of steroid-related pressure rise. Prior vitrectomy was among the exclusion criteria for this study. The SCORE Study pressure results were comparable to other analyses - Chuang et al found that $39 \%$ of eyes receiving $4 \mathrm{mg}$ IVTA for retinal vein occlusion developed secondary ocular hypertension. ${ }^{1}$

Vasconcelos-Santos et al recently found that $32 \%$ of 150 patients who received $4 \mathrm{mg}$ of IVTA in clinic, predominantly for macular degeneration, choroidal neovascular membranes, diabetic macular edema, and vascular occlusions, developed IOP $\geq 21 \mathrm{mmHg}$ postinjection. ${ }^{2}$ Of note, 17 eyes in that study were postvitrectomy, and that subgroup was associated with a lower peak IOP, although there was no significant difference in mean IOP, compared with the nonvitrectomized majority. Vitrectomized eyes were either excluded from or a smaller subgroup of each of these studies.

Animal studies indicate that vitrectomized eyes exhibit different pharmacokinetics than nonvitrectomized eyes. Schindler et al originally measured the disappearance of triamcinolone by colorimetric test after injection of $0.5 \mathrm{mg}$ into rabbit eyes. ${ }^{6}$ It cleared in 41 days in nonvitrectomized eyes, 16.8 days in vitrectomized eyes, and 6.5 days in eyes status postvitrectomy and lensectomy. Chin et al looked at the vitreous concentration of triamcinolone acetonide in vitrectomized and nonvitrectomized rabbit eyes at various times postinjection, and they found clearance to be faster in vitrectomized eyes, with a triamcinolone half-life of 1.57 days, compared to 2.89 in the nonvitrectomized group. ${ }^{5}$ In humans, Inoue et al examined the vitreous of twelve eyes undergoing vitrectomy with prior subtenon or intravitreal triamcinolone injection. ${ }^{18}$ Vitreous concentration was higher after intravitreal injection $(1.22 \mu \mathrm{g} / \mathrm{mL})$ than subtenon $(0.20 \mu \mathrm{g} / \mathrm{mL})$, and between the two with intravitreal injection in vitrectomized eyes.

The clinical rate of secondary elevation in intraocular pressure following IVTA in a vitrectomized eye has not been well described. Konstantinidis et al recently performed 23-gauge vitrectomy with a smaller dose (1 mg) of IVTA injected at the end of surgery for epiretinal membranes in 39 eyes. ${ }^{19}$ Thirteen percent of eyes developed IOP $\geq 21 \mathrm{mmHg}$ at postoperative month 1 and required initiation of glaucoma drops postoperatively, but the mean IOP did not differ statistically from baseline. Of note, that series' visual outcomes were favorable, compared with other studies of 23 -gauge vitrectomy for epiretinal membrane in the absence of adjunct triamcinolone.

Even the effect of vitrectomy itself on intraocular pressure remains unclear. Chang's LXII Edward Jackson lecture correlated vitrectomy with subsequent open-angle glaucoma, particularly in the absence of the crystalline lens. ${ }^{7}$ Koreen et al looked at incidence of secondary open-angle glaucoma after vitrectomy (without adjunctive IVTA) in 285 eyes. ${ }^{8}$ Open-angle glaucoma developed in $11.6 \%$ of patients in that study. Lens status was a significant variable, with only $1.4 \%$ of phakic eyes getting glaucoma, compared with $15 \%$ of nonphakic eyes. The study results suggest that the combination of lens removal and vitrectomy, in particular, is responsible for elevated pressure in a significant minority of eyes, possibly due, at least in part, to increased oxygenation in the anterior chamber angle.

Lastly, the type of triamcinolone injected may play a role in the risk profile. This series involved only preservative-free triamcinolone acetonide injectable suspension. Different triamcinolone suspensions, with and without preservative, may affect the drug's pharmacokinetic properties. Preservativefree triamcinolone acetonide has been observed to crystallize differently and aggregate and sink more in the vitreous than other formulations..$^{20,21}$ Most glaucoma-related studies of triamcinolone have involved older formulations, and it is not known whether the preparation itself has any role in the development of glaucoma or other effects.

In our series, all patients received vitrectomy, phacoemulsification, and intravitreal triamcinolone acetonide (4 mg) at the completion of surgery. Excluding postoperative day 1 , twelve of 131 eyes (9\%) developed IOP $\geq 25 \mathrm{mmHg}$ at some point in the postoperative period. Seven eyes (5\%) developed a rise in IOP $\geq 10 \mathrm{mmHg}$ from baseline. 
Six eyes $(5 \%)$ required either initiation or addition of glaucoma drops to their regimen postoperatively to control IOP. One year after surgery, three eyes continued to demonstrate elevated IOP and required multiple glaucoma drops. No eyes required surgery for steroid response or any sort of open-angle glaucoma. Secondary ocular hypertension in this study had a statistically significant correlation to a higher baseline IOP and to pre-existing glaucoma, but did not appear related to age, sex, presence of diabetes, or vitrectomy prior to the combined procedure.

To our knowledge, this is the first large review of the pressure effects of IVTA in the setting of combined vitrectomy and lens removal. It is of particular interest that the rate of postoperative glaucoma in our study appears lower than that of nonphakic patients undergoing vitrectomy in Koreen et al. ${ }^{8}$ If vitrectomy in the absence of a crystalline lens alters the functional properties of the angle via inflammatory changes, we propose that the IVTA may have a beneficial effect on angle function by modulating postoperative inflammation. The patients in this study had predominantly oncologic and radiation-related pathology, both of which might be expected to demonstrate more robust postoperative inflammation than more typical surgical pathology, such as epiretinal membranes and macular holes. The combination of vitrectomy with phacoemulsification likewise potentiates postoperative inflammation. The relatively low rate of secondary glaucoma in our study patients, compared to known rates of vitrectomy alone in nonphakic eyes, may be attributable to the adjunctive IVTA.

This study's limitations include its retrospective structure, lack of control arm, and extension from a single practitioner and his fellows. Because of the surgeon's oncologic practice in a tertiary care academic center, the eyes had a broad range of vitreoretinal pathology that is not reflective of the general retina community. Many of the operated eyes had extensive exudative and inflammatory components to their underlying disease, affecting the postoperative course and possibly the pharmacologic properties of the IVTA.

Our experience suggests that $4 \mathrm{mg}$ intravitreal triamcinolone acetonide injection at the end of combined vitrectomy and phacoemulsification surgery has an acceptable side effect profile, with few patients receiving glaucoma drops postoperatively, and no cases requiring surgical intervention for steroid-induced glaucoma. The reduced incidence of secondary ocular hypertension, compared to prior studies of IVTA in mostly or entirely nonvitrectomized eyes, likely reflects altered clearance of the medicine after removal of the vitreous. Our low incidence of secondary ocular hypertension, compared to prior analysis of the effects of vitrectomy alone, may even indicate a beneficial effect of IVTA on intraocular pressure in these patients. The consequences of intravitreal triamcinolone in the surgical setting deserve further study as the drug's therapeutic applications continue to expand.

\section{Disclosures}

The authors have no proprietary or commercial interest in any materials discussed in this article.

\section{References}

1. Chuang LH, Yeung L, Wang NK, Chen HS, Ku WC, Lai CC. Secondary ocular hypertension after intravitreal injection with $2 \mathrm{mg}$ or $4 \mathrm{mg}$ of triamcinolone in retinal vein occlusion. J Ocul Pharmacol Ther. 2010;26(4):325-328.

2. Vasconcelos-Santos DV, Nehemy PG, Schachat AP, Nehemy MB. Secondary ocular hypertension after intravitreal injection of $4 \mathrm{mg}$ of triamcinolone acetonide: incidence and risk factors. Retina. 2008;28(4):573-580.

3. Young S, Larkin G, Branley M, Lightman S. Safety and efficacy of intravitreal triamcinolone for cystoid macular oedema in uveitis. Clin Experiment Ophthalmol. 2001;29(1):2-6.

4. Wingate RJB, Beaumont PE. Intravitreal triamcinolone and elevated intraocular pressure. Aust NZJ Ophthalmol. 1999;27(6):431-432.

5. Chin H, Park T, Moon Y, Oh J. Difference in clearance of intravitreal triamcinolone acetonide between vitrectomized and nonvitrectomized eyes. Retina. 2005;25(5):556-560.

6. Schindler RH, Chandler D, Thresher R, Machemer R. The clearance of intravitreal triamcinolone acetonide. Am J Ophthalmol. 1982;93(4):415-417.

7. Chang S. LXII Edward Jackson lecture: open angle glaucoma after vitrectomy. Am J Ophthalmol. 2006;141(6):1033-1043.

8. Koreen L, Yoshida N, Escariao P, et al. Incidence of, risk factors for, and combined mechanism of late-onset open-angle glaucoma after vitrectomy. Retina. 2011;32(1):160-167.

9. Blankenship GW, Flynn HW Jr, Kokame GT. Posterior chamber intraocular lens insertion during pars plana lensectomy and vitrectomy for complications of proliferative diabetic retinopathy. Am J Ophthalmol. 1989;108(1):1-5.

10. Jun Z, Pavlovic S, Jacobi KW. Results of combined vitreoretinal surgery and phacoemulsification with intraocular lens implantation. Clin Experiment Ophthalmol. 2001;29(5):307-311.

11. Soheilian M, Mirdehqhan SA, Peyman GA. Sutureless combined 25 -gauge vitrectomy, phacoemulsification, and posterior chamber intraocular lens implantation for management of uveitic cataract associated with posterior segment disease. Retina. 2008;28(7):941-946.

12. Lee DY, Jeong HS, Sohn HJ, Nam DH. Combined 23-gauge sutureless vitrectomy and clear corneal phacoemulsification in patients with proliferative diabetic retinopathy. Retina. 2011;31(9):1753-1758.

13. Hikichi T, Kosaka S, Takami K, et al. 23- and 20-gauge vitrectomy with air tamponade with combined phacoemulsification for idiopathic macular hole: a single-surgeon study. Am J Ophthalmol. 2011;152(1): 114-121.

14. Enaida H, Hata Y, Ueno A, et al. Possible benefits of triamcinoloneassisted pars plana vitrectomy for retinal diseases. Retina. 2003;23(6): 764-770.

15. Bhisitkul RB, Winn BJ, Lee OT, et al. Neuroprotective effect of intravitreal triamcinolone acetonide against photoreceptor apoptosis in a rabbit model of subretinal hemorrhage. Invest Ophthalmol Vis Sci. 2008;49(9):4071-4077.

16. Ebrahem Q, Minamoto A, Hoppe G, Anand-Apte B, Sears JE. Triamcinolone acetonide inhibits IL-6 and VEGF-induced angiogenesis downstream of the IL-6 and VEGF receptors. Invest Ophthalmol Vis Sci. 2006;47(11):4935-4941. 
17. The SCORE Study Research Group. A randomized trial comparing the efficacy and safety of intravitreal triamcinolone with observation to treat vision loss associated with macular edema secondary to central retinal vein occlusion: the Standard Care vs Corticosteroid for Retinal Vein Occlusion (SCORE) Study Report 5. Arch Ophthalmol. 2009;127(9):1101-1114.

18. Inoue M, Takeda K, Morita K, Yamada M, Tanigawara Y, Oguchi Y. Vitreous concentrations of triamcinolone acetonide in human eyes after intravitreal or subtenon injection. Am J Ophthalmol. 2004;138(6):1046-1048.

19. Konstantinidis L, Gerguiga M, Beknazar E, Wolfensberger TJ. Anatomic and functional outcome after 23-gauge vitrectomy, peeling, and intravitreal triamcinolone for idiopathic macular epiretinal membrane. Retina. 2009;29(8):1119-1127.
20. Moshfeghi AA, Nugent AK, Nomoto H, Sanislo SR, Kitchens JW, Moshfeghi DM. Triamcinolone acetonide preparations: impact of crystal size on in vitro behavior. Retina. 2009;29(5):689-698.

21. Spitzer MS, Mlynczak T, Schultheiss M, et al. Preservative-free triamcinolone acetonide injectable suspension versus "traditional" triamcinolone preparations: impact of aggregate size on retinal biocompatibility. Retina. 2011;31(10):2050-2057.

\section{Publish your work in this journal}

Clinical Ophthalmology is an international, peer-reviewed journal covering all subspecialties within ophthalmology. Key topics include: Optometry; Visual science; Pharmacology and drug therapy in eye diseases; Basic Sciences; Primary and Secondary eye care; Patient Safety and Quality of Care Improvements. This journal is indexed on

Submit your manuscript here: http://www.dovepress.com/clinical-ophthalmology-journal

\section{Dovepress}

PubMed Central and CAS, and is the official journal of The Society of Clinical Ophthalmology (SCO). The manuscript management system is completely online and includes a very quick and fair peer-review system, which is all easy to use. Visit http://www.dovepress.com/ testimonials.php to read real quotes from published authors. 